\title{
RF AMPLIFIER CHOICE FOR THE ISAC SUPERCONDUCTING LINAC
}

\author{
I. Bylinskii, K. Fong, J. Lu, A.K. Mitra, C. Owen, TRIUMF, Vancouver, Canada
}

\section{Abstract}

A superconducting linac is being commissioned at TRIUMF as an extension to the existing room temperature accelerator of exotic ions at ISAC. It will increase the isotope final energy from 1.5 to $6.5 \mathrm{MeV} / \mathrm{u}$. Acceleration is accomplished in 40 bulk niobium quarter wave superconducting cavities operating at 106 and 141 $\mathrm{MHz}$. Each cavity is energized from an independent RF amplifier with power rating up to $1 \mathrm{~kW} \mathrm{cw}$. Both vacuum tube and solid-state amplifiers were considered as a viable option for the drivers. The paper compares many important parameters of these 2 amplifiers such as reliability, serviceability, capital and maintenance costs, as well as operating characteristics: gain linearity, phase noise, phase drift and others. Test results of prototypes of both types of amplifiers and 1-year operational experience of 20 tube amplifiers are discussed. Based on that the amplifier design recommendations are formulated.

\section{INTRODUCTION}

ISAC-2 superconducting (SC) linac will consist of 3 parts: low, medium, and high beta sections. The medium beta section was commissioned last year and comprises $20 \mathrm{SC}$ cavities driven by tube amplifiers at $106 \mathrm{MHz}$ [1]. The high beta section is being developed now; this will require another 20 power amplifiers (PA) to drive the SC cavities at $141 \mathrm{MHz}$.

\section{Beam Dynamics Requirements}

Accelerated beam parameters are largely dependent on the RF system performance. RF amplitude and phase instabilities and long term drifts affect beam energy stability, energy and time spread at the experimental station. RF control system plays fundamental role in stabilizing RF amplitudes and phases in each accelerating cavity. But RF controls has its own limitations in regulation of RF parameters in the superconducting cavities with extremely high $\mathrm{Q}$. In this conditions RF amplifier characteristics are of high importance in achieving desired RF field stability. Beam dynamics simulations suggest the following RF stability requirements per cavity:

$\begin{array}{ll}\text { Amplitude variations (rms) } & <0.2 \% \\ \text { Phase fluctuations (rms) } & <0.2^{\circ}\end{array}$

\section{SC Cavity Parameters}

ISAC-2 SC cavities feature extremely high Q: up to $10^{9}$ and thus very low RF losses. An accelerating gradient of $6 \mathrm{MV} / \mathrm{m}$ can be achieved with only 7 Watts of RF power dissipation in the cavity walls [2]. Beam load on the RF field is negligible because of very low intensity of radioactive ions. On the other hand high-Q load constitutes an extremely narrow operating frequency bandwidth: a fraction of a Hertz. In order to achieve stable
RF regulation the cavity is driven in an over-coupled regime. This reduces the loaded $\mathrm{Q}$ value and expands the operational bandwidth to about $20 \mathrm{~Hz}$. Corresponding coupling coefficient $\beta$ is $\approx 200$; and required incident $\mathrm{RF}$ power is about $200 \mathrm{~W}$.

\section{AMPLIFIER SPECIFICATIONS}

\section{PA Operation Modes}

ISAC accelerator operational mode is continuous wave (cw). PA will feed the RF cavity via a circulator with the cavity absorbing approximately $7 \mathrm{~W}$, and the rest of the $200 \mathrm{~W}$ of the incident power will be reflected back into the circulator load. In this mode the amplitude, phase and frequency are regulated with feedback loops.

In order to obtain high RF gradients the SC cavity has to be conditioned against field emission. It should be performed in a pulse mode to prevent rapid boiling of helium due to excessive heating of the SC cavity. During high voltage conditioning no RF regulation is applied, and significant fraction of RF power is consumed by emitted electrons, which are responsible for the degraded Q. Conditioning goal for the accelerated gradient is about 10 $\mathrm{MV} / \mathrm{m}$, which requires $600 \mathrm{~W}$ of $\mathrm{RF}$ incident power. Pulse mode is set at $50 \%$ duty cycle and 1 -second pulse duration.

\section{RF Amplifier Basic Parameters

$\begin{array}{ll}\text { Operating Frequency Range } & 141.0-142.0 \mathrm{MHz} \\ \text { CW Power Output } & 600 \mathrm{Watts} \\ \text { Maximum RF input power } & +5 \mathrm{dBm} \\ \text { Gain } & 55 \pm 2 \mathrm{~dB}\end{array}$ \\ Operating Load VSWR $\quad 1.01: 1$ to $1.5: 1$}

\section{Specific Amplifier Parameters}

A new RF control system has been optimized during medium beta SC linac commissioning and initial operation [3]. This experience has allowed us to set specific RF requirements represented in Table 1. Here the gain linearity and phase variation requirements apply to both short term power sweep as well as to long term drift, which can occur during amplifier warm up.

Table 1: Amplifier specific requirements

\begin{tabular}{|c|c|c|}
\hline Mode of operation & CW operation & Pulse conditioning \\
\hline Power range & $1-250$ Watt & $250-600$ Watt \\
\hline Gain linearity & $< \pm 0.5 \mathrm{~dB}$ & $< \pm 2.0 \mathrm{~dB}$ \\
\hline Phase drift/variation & $< \pm 5^{\circ}$ & $< \pm 20^{\circ}$ \\
\hline Phase noise*, rms & $0.3^{\circ}$ & _ \\
\hline Amplitude noise* & $0.6 \%$ & - \\
\hline
\end{tabular}




\section{TUBE AND SOLID STATE AMPLIFIERS COMPARISON}

Two choices were considered for the $141 \mathrm{MHz}$ amplifier: vacuum tube PA and solid state $(\mathrm{S} / \mathrm{S}) \mathrm{PA}$. In order to compare these 2 options we analyzed our experience with similar amplifiers being in use at ISAC. For the specific requirements we evaluated a few prototype amplifiers: four S/S PAs: 3 commercial units and one of TRIUMF design on one hand and a tube PA on the other hand.

\section{Prototype Amplifiers Measurements}

Gain and phase variation dependences on the output power for the amplifiers of each kind are presented in Figures 1 and 2. Amplifiers were running into $50 \mathrm{Ohm}$ dummy load, warmed up and stabilized. According to this test both type of amplifiers meet corresponding specifications. Figure 3 shows same amplifiers' phase noise in the condition of $250 \mathrm{~W}$ output loaded to the 50 Ohm. Tube amplifier generates phase noise, which exceeds the specification limit in two resonances: around $60 \mathrm{~Hz}$ (cooling fan mechanical vibration) and $120 \mathrm{~Hz}$ (DC power supply rectification ripple).

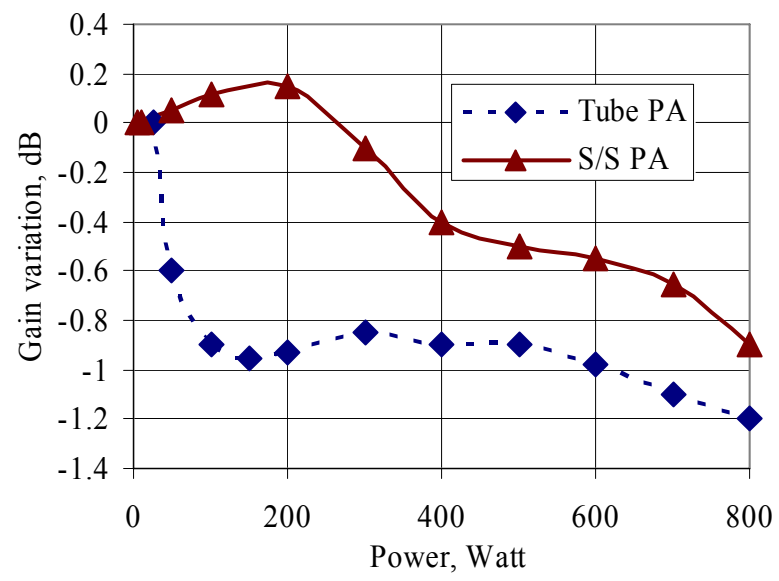

Figure 1: PA gain variation vs output power.

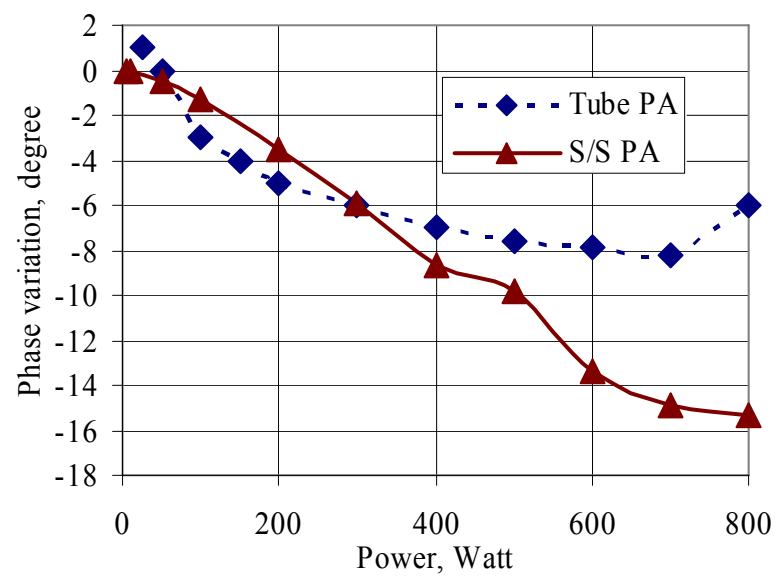

Figure 2: PA phase variation vs output power.
In addition to the stable condition measurements we also measured a phase drift in the transition from cold state to stable warm state with $250 \mathrm{~W}$ output loaded to the 50 $\mathrm{Ohm}$. The phase drift of the semiconductor amplifier was less than 2 degrees monitored during 6 hours of operation. The phase shift of the tube amplifier from the filament "off" state rose up to +13 degrees during first 15 minutes and then stabilized. From the filament "on" condition the phase drifted down to -25 degrees in about 4 minutes, then during 15 minutes gradually shifted back up to -15 degrees, and then stabilized. This represents a problem, because during transition the RF controls tuning is no longer optimal.

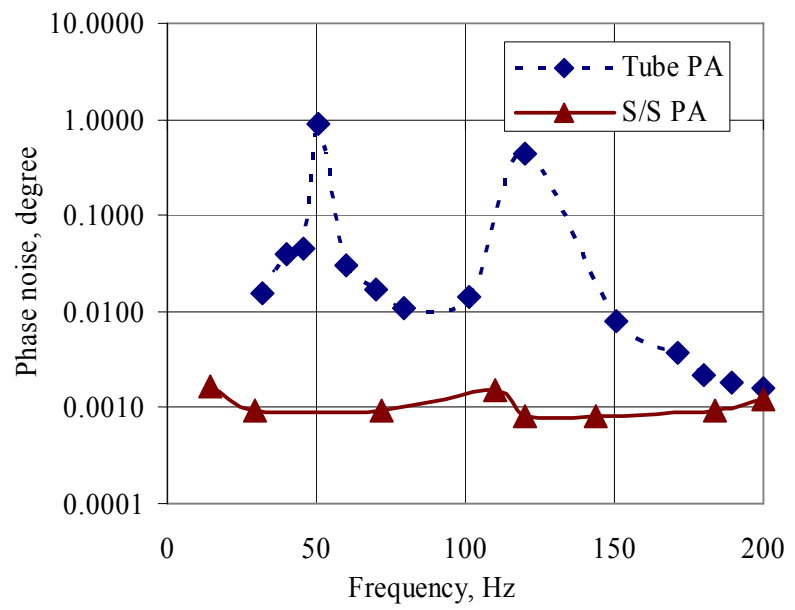

Figure 3: PA phase noise from 0 to $200 \mathrm{~Hz}$.

\section{Performance, Reliability and Serviceability}

Over last few years of development, commissioning and operation of the medium beta cavities we have accumulated sufficient experience about performance of the $106 \mathrm{MHz}, 800 \mathrm{~W}$ tube amplifiers [4]. Generally speaking, they have met our original specifications. Nevertheless we have seen a number of problems in their exploitation.

First of all - a phase noise issue. In the fist 4 prototype amplifiers a substantial phase noise was generated by the cooling fans due to vibration. Amplifier mechanical layout had to be changed to mitigate this issue. Nevertheless a marginal phase noise still remains present at the PA output due to power supply rectification ripple mentioned above. The remedies for this could be an improved DC filtering or application of a high frequency ( $>30 \mathrm{kHz}$ ) switching HV DC power supply.

Another issue we faced with the tube input impedance degradation and an output circuitry detuning. System appeared to be sensitive to the tube parameters change (ageing) and required adjustments when approaching tube lifetime. Table 2 represents PA parameters comparison for the new tube and after it was taken out of service.

Tube ageing itself manifested quite a painful problem. When superconducting cavity lost regulation in the middle of the long experimental run, the root cause of the failure was not always obvious for the operators. And there were a number of attempts to recover the operation 
by retuning the amplifier and RF controls before we realized that the tube could not deliver enough output power anymore. A solution to this problem could be found with monitoring of the tube amplification gain. Tube guaranteed lifetime is about 5000 hours. In our case all 20 tubes operated over 8700, after which their performance degraded and they had to be replaced.

Table 2: Aged tube amplifier parameters degradation

\begin{tabular}{|c|c|c|}
\hline Parameter & New tube & Retired tube \\
\hline Tube input VSWR & 1.2 & 2.5 \\
\hline Tube stage gain & $14 \mathrm{~dB}$ & $2 \mathrm{~dB}$ \\
\hline Maximum power & $900 \mathrm{~W}$ & $90 \mathrm{~W}$ \\
\hline Frequency & $106.1 \mathrm{MHz}$ & $109.5 \mathrm{MHz}$ \\
\hline Phase shift & 0 & $>100^{\circ}$ \\
\hline
\end{tabular}

On the other hand the ISAC-1 room temperature linac utilizes more then 20 semiconductor PAs rated from 300 to 2000 Watts. Most of them serve as a tube amplifier driver; others are the final PAs for the small buncher and chopper cavities. Half of the amplifiers are commercial units; the other half is developed at TRIUMF. There were about 50 failures of S/S PA since beginning of ISAC-1 operation ( 7 to 10 years). Almost all the break-downs happened to the poorly protected or unprotected power transistors. Transistors with protection circuits have been working fail-free. High voltage transients and input overdrive from RF controls were responsible for transistors damage.

Troubleshooting and servicing of both tube and semiconductor amplifiers of this size are relatively simple, especially if design is modular and leaves enough room for easy access to the components, and if sufficient diagnostics is incorporated into amplifier design. Obviously for the tube amplifier one has to take all the safety precautions dealing with high voltage, which is not the case for the semiconductor PA.

\section{Capital and maintenance costs evaluation}

The following Table 3 compares the total cost of purchasing and operating of one tube PA and one Solid State PA over 10 years, assuming purchase quantity of 20 .

Table 3: PA capital and operation cost

\begin{tabular}{|l|c|c|}
\hline Description & Tube PA & S/S PA \\
\hline Budgetary price & $15 \mathrm{k} \$$ & $25 \mathrm{k} \$$ \\
\hline Electricity cost & $3 \mathrm{k} \$$ & $2 \mathrm{k} \$$ \\
\hline Tube / transistor replacement & $10 \mathrm{k} \$$ & $0.1 \mathrm{k} \$$ \\
\hline Fan \& other components replacement & $1 \mathrm{k} \$$ & $1 \mathrm{k} \$$ \\
\hline Total cost over 10 years & $29 \mathrm{k} \$$ & $28.1 \mathrm{k} \$$ \\
\hline
\end{tabular}

We account that tube replacement would be required every 12 months whereas transistor might need to be replaced once in 10 years. For a tube amplifier, the electricity cost is higher because its efficiency is lower.

\section{Summary}

Table 4 represents a comparison of advantages and disadvantages of tube PA and S/S PA for the SC linac application.

Table 4: Tube and S/S PA comparison

\begin{tabular}{|c|c|c|}
\hline Description & Tube PA & S/S PA \\
\hline Class of Operation & Class A & Class AB \\
\hline Performance & $\begin{array}{c}\text { Satisfies most of } \\
\text { requirements; but phase } \\
\text { drift \& noise are marginal }\end{array}$ & $\begin{array}{c}\text { Meets all } \\
\text { requirements }\end{array}$ \\
\hline Reliability & $\begin{array}{c}\text { Tube is rugged, but needs } \\
\text { replacement every } 12 \\
\text { months }\end{array}$ & $\begin{array}{l}\text { Transistor } \\
\text { failure is rare, } \\
\text { if protected }\end{array}$ \\
\hline Serviceability & Reasonable & Reasonable \\
\hline System Downtime & $\begin{array}{c}\text { Possible interruptions due } \\
\text { to tube ageing }\end{array}$ & Very little \\
\hline Failure Diagnosis & $\begin{array}{c}\text { Easy with incorporated } \\
\text { diagnostics }\end{array}$ & $\begin{array}{c}\text { Easy with } \\
\text { incorporated } \\
\text { diagnostics }\end{array}$ \\
\hline High Voltage & $3.5 \mathrm{kV}$ & Below $50 \mathrm{~V}$ \\
\hline Efficiency at $200 \mathrm{~W}$ & $\sim 25 \%$ & $\sim 35 \%$ \\
\hline Maintenance cost & 1,000 \$/year & 100 \$/year \\
\hline
\end{tabular}

\section{S/S PA DESIGN RECOMMENDATIONS}

The following are the recommendations for design of a semiconductor PA for the SC linac, aiming reliable operation and easy service.

- Drain voltage and current detection and fast DC cut off

- Output VSWR protection

- Adequate filtering against RF leakage for DC PS

- Monitoring of DC voltage, current and temperature

- Air cooling

\section{CONCLUSION}

The semiconductor PA is better in a few critical parameters compared with the tube PA for SC linac application. The only disadvantage of the $\mathrm{S} / \mathrm{S} \mathrm{PA}$ is a higher capital cost, which will be offset by much lower maintenance cost in a long run. For these reasons TRIUMF has chosen a semiconductor amplifier for the high beta section of the ISAC-2 linac.

\section{REFERENCES}

[1] R.E. Laxdal et al., "Initial Commissioning Results from the ISAC-II SC Linac", LINAC'06, Knoxville, August 2006, p. 521.

[2] R.E. Laxdal et al., "Recent progress in the superconducting RF Program at TRIUMF/ISAC", Physica C 441 (2006), pp. 13-20.

[3] K. Fong, M. Laverty and S. Fang, "Status of RF Control System for ISAC-II Superconducting Cavities”, LINAC'04, Lübeck, August 2004, p. 450.

[4] A.K. Mitra et al., "RF Amplifiers and Structures for ISAC/TRIUMF",APAC2007, RRCAT, Indore, India, January 29-February 2, 2007. 